\title{
First Report of Compartment Syndrome and Myonecrosis in a Patient with Hemoglobin SC Disease
}

\author{
Muntadhar Al Moosawi ${ }^{1}$ and Hatoon Ezzat ${ }^{2}$ \\ ${ }^{1}$ The University of British Columbia \\ ${ }^{2}$ Providence Hematology, St Paul's Hospital
}

November 16, 2020

\begin{abstract}
Myonecrosis and compartment syndrome are rarely seen in sickle cell disease (SCD). Myonecrosis and compartment syndrome have not been previously reported in HbSC. This case report presents the first case of an HbSC patient presenting to emergency department (ED) with bilateral leg pain and swelling.
\end{abstract}

\section{Introduction}

Sickle cell anemia (SCA) is an inherited blood disorder caused by a single nucleotide substitution (A to T) in the codon of the sixth amino acid at the $\beta$-globin gene $(H B B)$ in chromosome 11 , resulting in a conversion of glutamic acid residue to a valine residue (Glu6Val). Consequently, the mutation leads to the synthesis of the well-known abnormal and unstable hemoglobin $\mathrm{S}(\mathrm{HbS})$. The substitution of a glutamic acid with lysine at the same position of the $\beta$-globin gene results in the formation of hemoglobin $\mathrm{C}(\mathrm{HbC})$, which is a milder variant of sickle cell disease. Hemoglobin SC disease occurs as a result of the co-inheritance of one HbS allele and another $\mathrm{HbC}$ allele ${ }^{1}$. In the US, HbSC accounts for nearly $30 \%$ of SCD patients with an incidence of 1 in every 6,173 newborns ${ }^{2}$. However, the disease is more prevalent in parts of West Africa, where HbSC accounts for more than $50 \%$ of $\mathrm{SCD}^{3}$.

HbSC disease has unique pathophysiological characteristics. The proportions of $\mathrm{HbS}$ and $\mathrm{HbC}$ in the red blood cells (RBCs) of HbSC patients is roughly equal ${ }^{4}$. Due to alterations of RBC membrane, HbC has a particular tendency to crystallization and loss of $\mathrm{K}^{+}$and water, causing intracellular dehydration, which leads to an increase in $\mathrm{HbS}$ polymerization in the cytoplasm of $\mathrm{HbSC}$ cells. This biochemical process is the main reason for the clinical manifestation of HbSC patients ${ }^{5}$.

Hemolysis is less intense in HbSC disease as compared to homozygous HbSS, but the presence of mild hemolysis results in mild chronic anemia. Nevertheless, the mean hemoglobin concentration in patients with HbSC tends to be higher than that of HbSS, and the erythrocyte lifespan is twice that of HbSS (29 vs. 15 days) with the RBC indices indicating low mean cell volume (MCV) in most cases ${ }^{6}$. The blood film shows characteristic findings including boat-shaped cells, and sometimes cells with straight-edged, six-sided hemoglobin $\mathrm{C}$ crystals can be seen. Characteristic HbSC poikilocytes can also present in the blood film along with numerous target cells, but irreversibly sickled cells are rare.

The clinical phenotype of patients with $\mathrm{HbSC}$ is unique, with painful vaso-occlusive crises being less frequent compared to SCA. However, proliferative retinopathy is seen more commonly ${ }^{7}$.

Myonecrosis has not been previously described in patient with $\mathrm{HbSC}$, and in the field of hemoglobinopathy; only 14 cases of myonecrosis are reported in the literature, all of which occurred in patients with HbSS. In SCD, this phenomenon is believed to be caused by muscle hypoxia due to vaso-occlusion by irreversibly 
sickled RBCs. On the other hand, muscle hypoxia leading to exertional rhabdomyolysis has been well described in patients with sickle cell trait (SCT). In a retrospective cohort study of approximately 48 thousand soldiers in the U.S army, individuals with SCT had $54 \%$ higher risk of developing rhabdomyolysis compared to individuals with normal genotype ${ }^{8}$. This potentially lethal complication is caused by severe breakdown of skeletal-muscle tissue that is precipitated by strenuous physical exertion, leading to systemic manifestations ${ }^{9}$. Furthermore, analysis of post activity changes in SCT individuals showed marginally diminished RBC deformability, hyperviscosity and increased concentration of circulating adhesion molecules ${ }^{10}$. The alteration in vascular cell adhesion mechanisms and the vascular endothelium damage in SCT carriers, if extensive, can affect muscle microcirculation, causing ischemia and rhabdomyolysis. Hyperkalemia as a result of the release of intracellular potassium is suspected to cause cardiac arrhythmias and death ${ }^{11-13}$.

We report a case of a 20-year old male who is known to have HbSC disease with mild clinical phenotype presenting to ED with bilateral leg swelling and pain that was more pronounced in the left leg, and was associated with numbness and weakness of mainly the distal part of the left foot. The patient was eventually diagnosed with non-traumatic compartment syndrome and myonecrosis.

Using EMBASE and MEDLINE databases, we found no previous reports of compartment syndrome and myonecrosis in patients with HbSC disease. Hence, this is the first report of this condition in this patient population.

\section{Case report}

\section{History and physical examination}

This is a 20-year old male with a known history of hemoglobin SC disease and a previous non-traumatic compartment syndrome involving the anterior compartment of the right leg, which resolved spontaneously without medical or surgical intervention. This incident resulted in mild sensory loss of the first webspace of the right foot. Approximately two years after the first episode, the patient presented at the ED after a sudden onset of bilateral shin pain that was more pronounced at the mediolateral aspect of the left shin and was accompanied by weakness and decreased sensation in the distal part of the left foot. The pain in the right leg subsided before the patient's arrival at ED.

The patient had a mild clinical phenotype with fewer than five episodes of painful vaso-occlusive crises during his lifetime and no other sickle cell complications. The left leg was swollen, tense, and tender with power of 1 to 2 of 5 in dorsiflexion, 2 of 5 plantar flexion, 2 of 5 inversion, and 2 of 5 eversion. A decreased light touch, pinprick, and temperature sensation was noted in the first webspace of the left foot. The pressure measurement in the anterior compartment of the left foot was over $125 \mathrm{mmHg}$ (normal is $<10 \mathrm{mmHg}$ ).

\section{Investigations and clinical management}

Microcytic anemia was noted in the complete blood count (CBC) with hemoglobin just below the patient's baseline ( $\mathrm{Hb} 103 \mathrm{~g} / \mathrm{L}, \mathrm{MCV} 66 \mathrm{fL}$ ) and signs of hemolysis explained by the patient's baseline of mild chronic hemolysis (reticulocyte count $170 \times 10^{9} / \mathrm{L}$, haptoglobin $<0.07 \mathrm{~g} / \mathrm{L}$ (normal is $0.3-2.0$ ), lactate dehydrogenase $373 \mathrm{U} / \mathrm{L}$ (normal is 115-230)). High-performance liquid chromatography (HPLC) was done 3 weeks before the incident and demonstrated the presence of $\mathrm{HbS}$ at $47 \%$, $\mathrm{HbC}$ at $45 \%$, and a small proportion of $\mathrm{HbF}$ accounting for only $1.5 \%$. Creatine kinase (CK) was elevated at $4,955 \mathrm{U} / \mathrm{L}$ at presentation (normal is $<165$ $\mathrm{U} / \mathrm{L}$ ), suggesting underlying rhabdomyolysis. An urgent magnetic resonance imaging (MRI) of the left leg demonstrated findings in favour of rhabdomyolysis and myonecrosis with signs of compartment syndrome (Figure 1) warranting urgent surgical intervention. Successful and uncomplicated left anterior and lateral compartment fasciotomies were performed (Figure 2) and a tissue from the left anterior tibialis muscle confirmed the diagnosis of myonecrosis (Figure 3). The wound was allowed to heal for 16 days after which a fasciotomy closure using a split-thickness skin graft was performed.

Over the course of three weeks of hospital admission, creatine kinase significantly increased, reaching a peak of 22,000 U/L on the third day of admission and then decreased steadily and normalized over a week. The kidney functions were overall preserved, except for a transient mild acute kidney injury that lasted 
approximately 5 days. Only one unit of packed red cells was transfused over the course of hospitalization as a pre-operative preparation when the hemoglobin was $73 \mathrm{~g} / \mathrm{L}$. Aggressive hydration and pain management were the mainstay of treatment and were maintained until patient's full recovery.

\section{Discussion}

Myonecrosis and compartment syndrome are known but rare complications of SCA. To date, there have been 15 reported cases of sickle cell myonecrosis, including this case. All of the previously reported cases occurred in patients with HbSS genotype. The clinical presentation of this case is consistent with that of HbSS cases in many ways. The pain and neurologic symptoms are characteristic of muscle and nerve injury seen in HbSS cases with similar complication. Although presented with bilateral symptoms, the condition involved the patient's left lower limb, particularly the anterior and lateral compartments of the left lower leg. However, this problem has occurred in the deltoid and quadriceps muscle groups in some of the reported cases $^{14}$. Creatine kinase was significantly elevated, a finding that is not usually observed in myonecrosis and usually indicates an underlying rhabdomyolysis, which was the case in our patient. Tageja et al. reported only three patients with elevated CK levels ${ }^{14}$. MRI is a key diagnostic tool in cases of myonecrosis with characteristic findings observed in the form of enhancement of $\mathrm{T} 1$ images, and an increased signal intensity of T2-weighed images is observed ${ }^{15}$. A muscle biopsy is the confirmatory diagnostic procedure and because the muscle necrosis can be focal and involve a small area, a large biopsy is necessary to make the diagnosis.

No standards are available for the management of myonecrosis in this group of patients, given the rarity of this condition. However, compartment syndrome generally requires urgent surgical intervention. Four weeks after the patient's presentation, an electromyogram revealed a significant injury to the left deep peroneal nerve, although the superficial peroneal nerve was spared for the most part. The condition was debilitating, but with the support of physiotherapy, the patient slowly regained mobility and was able to walk independently.

\section{Conclusion}

Myonecrosis has been previously reported in patients with HbSS. This is the first report of this condition in HbSC disease. The mechanism and pathophysiology of this condition are not very well understood in this group of patients. Prompt diagnosis and management is warranted to prevent long-term debilitation. More research is required in this area to help further understand the pathophysiology of myonecrosis in patients with SCD.

Conflict of interest: The authors declare that they have no conflict of interest.

Acknowledgement : We thank the patient for his participation in this report

\section{References:}

1. Pecker LH, Schaefer BA, Luchtman-Jones L. Knowledge insufficient: the management of haemoglobin SC disease. Br J Haematol. 2017;176(4):515-26.

2. Therrell BL Jr, Lloyd-Puryear MA, Eckman JR, Mann MY. Newborn screening for sickle cell diseases in the United States: A review of data spanning 2 decades. Semin Perinatol. 2015;39(3):238-51.

3. Saraf SL, Molokie RE, Nouraie M, Sable CA, Luchtman-Jones L, Ensing GJ, et al. Differences in the clinical and genotypic presentation of sickle cell disease around the world. Paediatr Respir Rev. 2014;15(1):412 .

4. Gualandro SFM, Fonseca GHH, Yokomizo IK, Gualandro DM, Suganuma LM. Cohort study of adult patients with haemoglobin SC disease: clinical characteristics and predictors of mortality. Br J Haematol. $2015 ; 171(4): 631-7$.

5. Nagel RL, Fabry ME, Steinberg MH. The paradox of hemoglobin SC disease. Blood Rev. 2003;17(3):16778. 
6. McCurdy PR, Sherman AS. Irreversibly sickled cells and red cell survival in sickle cell anemia: a study with both DF32P and 51CR. Am J Med. 1978;64(2):253-8.

7. Lawrence C, Fabry ME, Nagel RL. The unique red cell heterogeneity of SC disease: crystal formation, dense reticulocytes, and unusual morphology. Blood. 1991;78(8):2104-12.

8. Nelson DA, Deuster PA, Carter R 3rd, Hill OT, Wolcott VL, Kurina LM. Sickle cell trait, rhabdomyolysis, and mortality among U.s. army soldiers. N Engl J Med. 2016;375(5):435-42.

9. Ferster K, Randy Eichner E. Exertional sickling deaths in army recruits with sickle cell trait. Mil Med. 2012;177(1):56-9.

10. Tripette J, Connes P, Beltan E, Chalabi T, Marlin L, Chout R, et al. Red blood cell deformability and aggregation, cell adhesion molecules, oxidative stress and nitric oxide markers after a short term, submaximal, exercise in sickle cell trait carriers. Clin Hemorheol Microcirc. 2010;45(1):39-52.

11. Loosemore M, Walsh SB, Morris E, Stewart G, Porter JB, Montgomery H. Sudden exertional death in sickle cell trait: Figure 1. Br J Sports Med. 2012;46(5):312-4.

12. Connes P, Frank S, Martin C, Shin S, Aufradet E, Sunoo S, et al. New fundamental and applied mechanisms in exercise hemorheology. Clin Hemorheol Microcirc. 2010;45(2-4):131-41.

13. Bergeron MF, Cannon JG, Hall EL, Kutlar A. Erythrocyte sickling during exercise and thermal stress. Clin J Sport Med. 2004;14(6):354-6.

14. Tageja N, Racovan M, Valent J, Zonder J. Myonecrosis in sickle cell anemia-overlooked and underdiagnosed. Case Rep Med. 2010;2010:659031.

15. Vicari P, Achkar R, Oliveira KRB, Miszpupten ML, Fernandes ARC, Figueiredo MS, et al. Myonecrosis in sickle cell anemia: case report and review of the literature. South Med J. 2004;97(9):894-6.

\section{Figure legends}

Figure 1. MRI; fluid sensitive sequence showing pathological high signal consistent with tissue edema. Low signal on $\mathrm{T} 1$ and mild hyperintensity on $\mathrm{T} 2$ weighed images were also observed.

Figure 2. Fasciotomy wound was allowed to heal with support of vacuum dressing.

Figure 3. Anterior tibialis muscle biopsy demonstrating muscle necrosis and inflammation; H\&E stain; original magnification $\mathrm{x} 40$. 

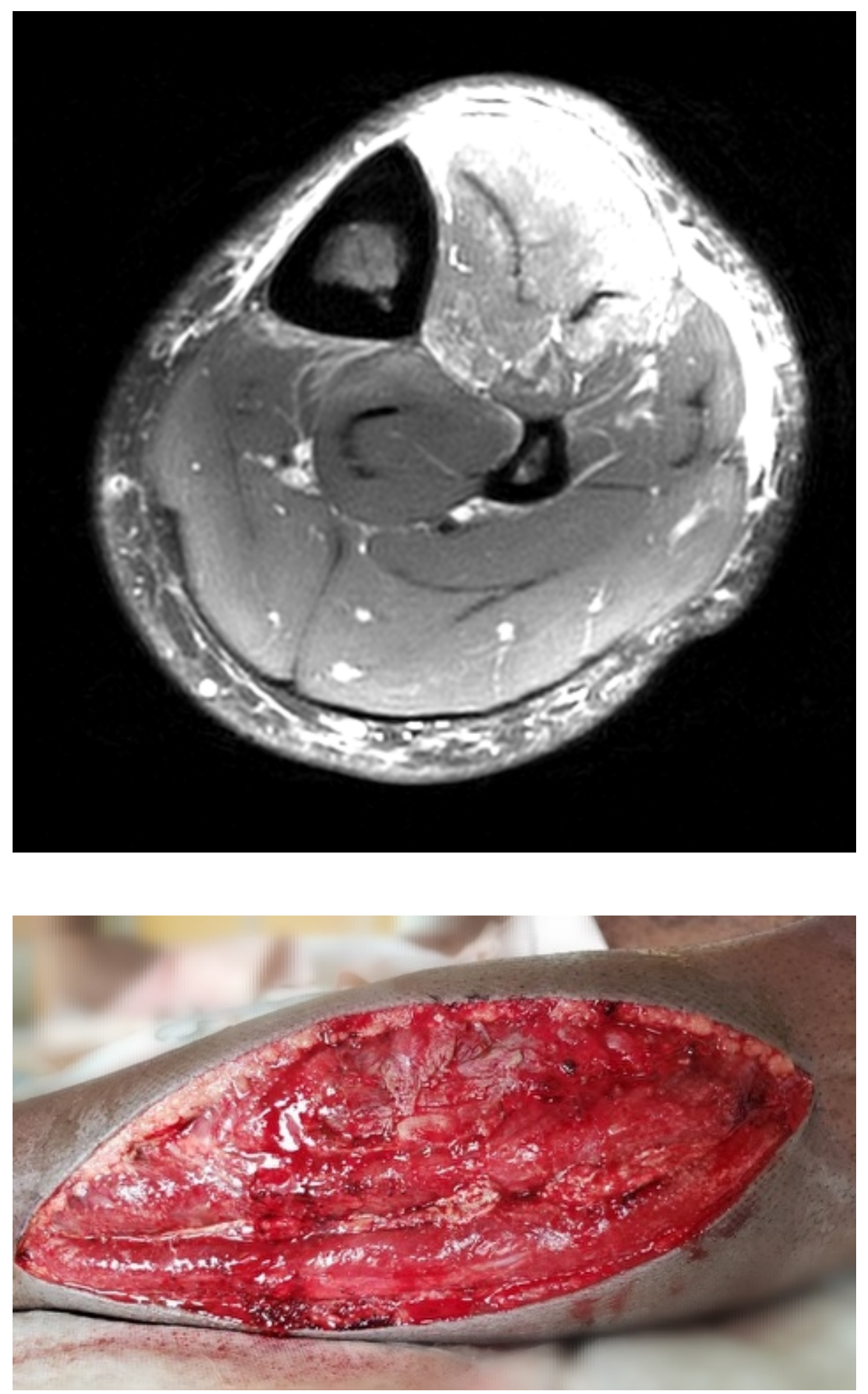


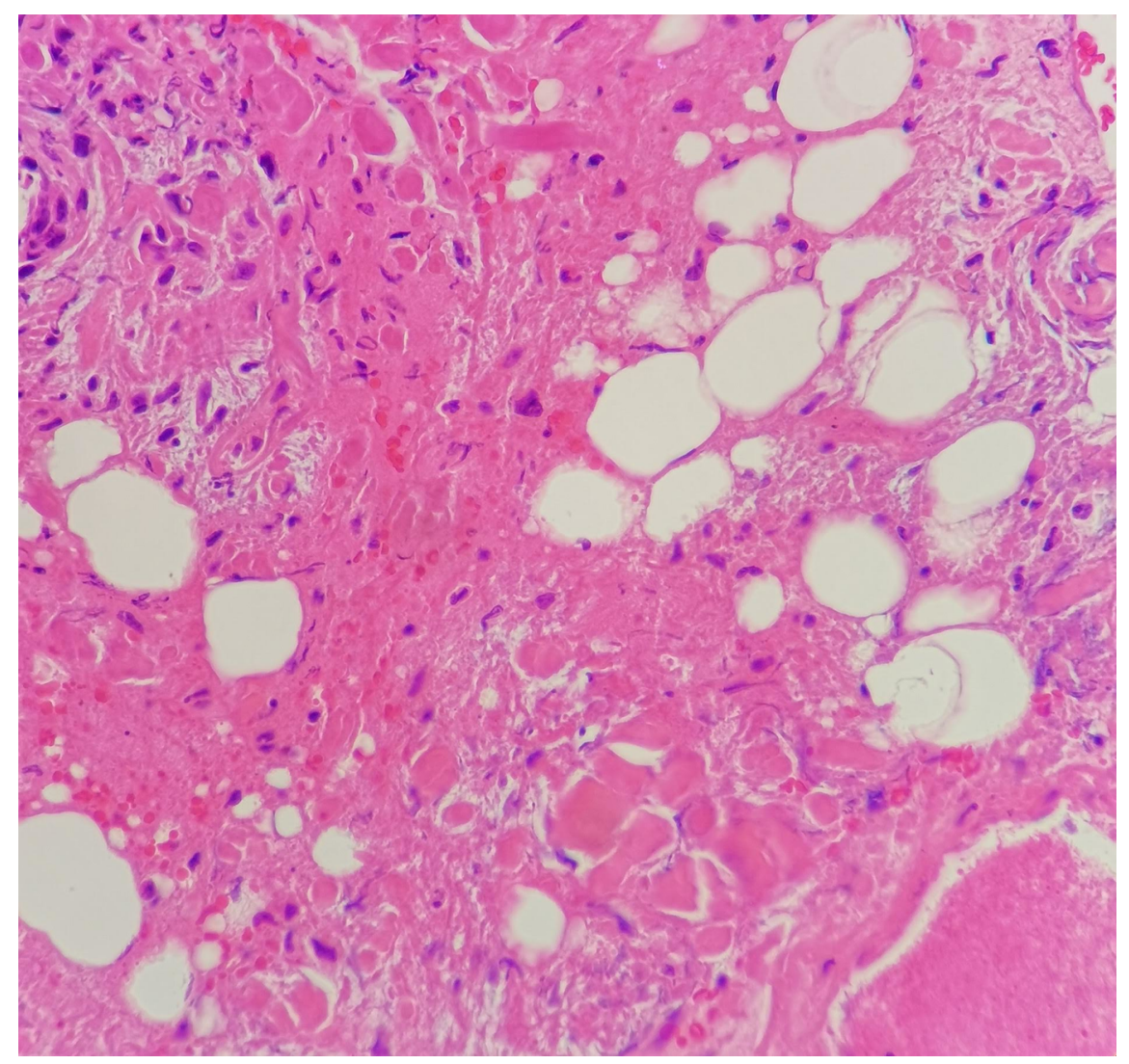

